\title{
Isolation and Polyphasic Characterization of Desulfuromonas versatilis sp. Nov., an Electrogenic Bacteria Capable of Versatile Metabolism Isolated from a Graphene Oxide-Reducing Enrichment Culture
}

\author{
Li Xie ${ }^{1,+}\left(\mathbb{D}\right.$, Naoko Yoshida ${ }^{1, *}++^{\mathbb{D}}$, Shun'ichi Ishii ${ }^{2}(\mathbb{D})$ and Lingyu Meng ${ }^{1}(\mathbb{D})$ \\ 1 Department of Civil Engineering, Nagoya Institute of Technology (Nitech), Nagoya 466-8555, Aichi, Japan; \\ xie705049124@126.com (L.X.); meng.lingyu@nitech.ac.jp (L.M.) \\ 2 Institute for Extra-Cutting-Edge Science and Technology Avant-Garde Research (X-Star), Japan Agency for \\ Marine-Earth Science and Technology (JAMSTEC), Yokosuka 237-0061, Kanagawa, Japan; sishii@jamstec.go.jp \\ * Correspondence: yoshida.naoko@nitech.ac.jp; Tel.: +81-527-355-437 \\ + These authors have contributed equally to this work and share first authorship.
}

Citation: Xie, L.; Yoshida, N.; Ishii, S.; Meng, L. Isolation and Polyphasic Characterization of Desulfuromonas versatilis sp. Nov., an Electrogenic Bacteria Capable of Versatile

Metabolism Isolated from a Graphene Oxide-Reducing Enrichment Culture. Microorganisms 2021, 9, 1953. https://doi.org/10.3390/ microorganisms 9091953

Academic Editors: Annette Rowe and Akihiro Okamoto

Received: 10 July 2021

Accepted: 9 September 2021

Published: 14 September 2021

Publisher's Note: MDPI stays neutral with regard to jurisdictional claims in published maps and institutional affiliations.

Copyright: (c) 2021 by the authors. Licensee MDPI, Basel, Switzerland. This article is an open access article distributed under the terms and conditions of the Creative Commons Attribution (CC BY) license (https:/ / creativecommons.org/licenses/by/ $4.0 /)$.
Abstract: In this study, a novel electrogenic bacterium denoted as strain NIT-T3 of the genus Desulfuromonas was isolated from a graphene-oxide-reducing enrichment culture that was originally obtained from a mixture of seawater and coastal sand. Strain NIT-T3 utilized hydrogen and various organic acids as electron donors and exhibited respiration using electrodes, ferric iron, nitrate, and elemental sulfur. The strain contained C16:1 $\omega 7 \mathrm{c}, \mathrm{C} 16: 0$, and C15:0 as major fatty acids and MK-8, 9, and 7 as the major respiratory quinones. Strain NIT-T3 contained four 16S rRNA genes and showed $95.7 \%$ similarity to Desulfuromonas michiganensis $\mathrm{BB}^{\mathrm{T}}$, the closest relative. The genome was $4.7 \mathrm{Mbp}$ in size and encoded 76 putative $c$-type cytochromes, which included 6 unique $c$-type cytochromes $(<40 \%$ identity) compared to those in the database. Based on the physiological and genetic uniqueness, and wide metabolic capability, strain NIT-T3 is proposed as a type strain of 'Desulfuromonas versatilis' sp. nov.

Keywords: Desulfuromonas; electrogenic bacteria; nitrate respiration; graphene oxide

\section{Introduction}

Anaerobic and extracellular electron-transferring (EET) bacteria are ubiquitously involved in the redox flow via solid conductors. Geobacter and Shewanella have been extensively studied for their functional roles in terrestrial and marine subsurface, whose anoxic environments are deficient in soluble electron acceptors. The unique metabolism of such bacteria has been applied in bioelectrochemical systems (BESs) to be used for wastewater treatment [1], production of renewable energy and value-added products [2], and bioremediation [3]. Both culture-dependent and -independent studies have revealed the presence and functional role of electrogenic microbes other than Geobacter and Shewanella [4]. Various factors, such as availability of the electron donor [5], the electric potential of a solid conductor [6], and the origin, affect the microbial composition in the system. Additionally, the surface chemistry of electrodes provides selective pressure during the early growth of the biofilm [7].

The genus Desulfuromonas has received much attention as a common electrogenic bacteria present in BESs [8-10]. Desulfuromonas species are found in natural environments, such as aquifers [11], sediment cores [12], and terrestrial mud volcanoes [13], suggesting a wide distribution. The genus Desulfuromonas consists of anaerobic chemoheterotrophs and was first proposed after the isolation of an elemental sulfur-reducing bacterium, Desulfuromonas acetoxidans DSM 684 ${ }^{\mathrm{T}}$, from anaerobic sulfide-containing marine 
mud [14]. At present, D. acetoxidans DSM $684^{\mathrm{T}}$ and seven other valid species have been proposed. D. acetexigens $2873^{\mathrm{T}}$ [15], Desulfuromonas palmitatis SDBY1 ${ }^{\mathrm{T}}$ [16], Desulfuromonas thiophila $\mathrm{NZ27}^{\mathrm{T}}$ [17], Desulfuromonas chloroethenica $\mathrm{TT}^{\mathrm{B}} \mathrm{B}^{\mathrm{T}}$ [18], Desulfuromonas michiganensis BB1 ${ }^{\mathrm{T}}$ [19], Desulfuromonas svalbardensis $112^{\mathrm{T}}$ [20], and Desulfuromonas carbonis $\mathrm{ICBM}^{\mathrm{T}}$ [21] have been isolated from anoxic freshwater sediments, marine sediment, freshwater mud, freshwater sediment, pristine river sediment, Arctic marine sediments, and coal-bed water, respectively. Recently, additional strains including Desulfuromonas sp. TF [22], Desulfuromonas sp. AOP6 [23], Desulfuromonas sp. TZ1 [24], and 'Desulfuromonas soudanensis' WTL [25] have been isolated from tidal flat sediment, sub-seafloor sediment, marine sediments, and anoxic deep subsurface brine, respectively.

All valid species of Desulfuromonas grow anaerobically and reduce sulfur, and exhibit iron respiration coupled with acetate oxidation; however, they are unable to respire using soluble nitrogenous and sulfuric compounds. The respiration specific to solid minerals and the presence of Desulfuromonas species in BESs suggest the substantial involvement of Desulfuromonas in electrode-driven metabolism, although such activity has been rarely proven in pure cultures. None of the valid species, except for two strains including Desulfuromonas sp. TZ1 [24] and 'D. soudanensis' WTL [25], generate an electric current in pure cultures, and both have been isolated from electrodes set up in environments, such as marine sediment and Soudan mine, respectively. These results suggest that Desulfuromonas species play substantial roles similar to those of two representative electrogenic genera, Geobacter and Shewanella, in microbial elemental cycling on solid conductors, especially in marine sediments.

Presently, the genomes of two strains, D. soudanensis WTL [25] and Desulfuromonas sp. AOP6 [23], have been published, and the genome of another strain, DDH964, is unpublished but uploaded on the National Center for Biotechnology Information (NCBI) database under the accession number CPO15080. The genomes are 1.64-4.40 Mb in size, contain $37.9-65.9 \%$ of broad G + C content, and have 2181-3924 coding sequences (CDSs). All three genomes encode a complete TCA cycle, a non-oxidative pentose phosphate pathway, Embden-Meyerhof-Parnas glycolysis/gluconeogenesis, and abundant CDSs associated with $c$-type cytochrome biosynthesis. The number of putative multiheme $c$-type cytochromes ranges from 37-44, and the number is comparable to those in the genera Geobacter and Shewanella. Additionally, genes involved in the biosynthesis of type IV pili, known to be involved in the formation of conductive biofilm [26], are commonly present in the three genomes.

In this study, a novel electrogenic bacterium of the genus Desulfuromonas, 'Desulfuromonas versatilis' NIT-T3 was isolated from an enrichment culture of graphene oxidereducing bacteria (GORB) that were initially obtained from a mixture of seawater and coastal sand [27]. GORB have been applied in the formation of hydrogel electrodes that generate electricity using synthetic medium [28], soil [29], and wastewater [30-32]. Physiological and genomic analysis of strain NIT-T3 revealed versatile metabolism, and the findings expand the understanding of the metabolism in the genus Desulfuromonas and its ecology in natural and artificial environments.

\section{Materials and Methods}

\subsection{Isolation and Growth Conditions}

Strain NIT-T3 was isolated from an enrichment culture of GORB (CS culture) that was obtained from a mixture of seawater and coastal sand, as described previously [27]. A DS-basal medium used for the isolation and growth of the strain contains $20 \mathrm{~g} / \mathrm{L}$ $\mathrm{NaCl}, 0.3 \mathrm{~g} / \mathrm{L} \mathrm{KCl}, 0.5 \mathrm{~g} / \mathrm{L} \mathrm{NH}_{4} \mathrm{Cl}, 0.1 \mathrm{~g} / \mathrm{L} \mathrm{CaCl} 2 \cdot 2 \mathrm{H}_{2} \mathrm{O}, 4 \mathrm{~g} / \mathrm{L} \mathrm{MgCl}_{2} \cdot 6 \mathrm{H}_{2} \mathrm{O}, 0.6 \mathrm{~g} / \mathrm{L}$ $\mathrm{KH}_{2} \mathrm{PO}_{4}, 2.5 \mathrm{~g} / \mathrm{L} \mathrm{NaHCO} 3,1 \mathrm{~mL} / \mathrm{L} \mathrm{SL}-10,1 \mathrm{~mL} / \mathrm{L} \mathrm{Se} / \mathrm{W}$ solution, vitamin-solution, and $0.2 \mathrm{mg} / \mathrm{L}$ resazurin and the basal medium was prepared anaerobically under flashing $\mathrm{N}_{2}: \mathrm{CO}_{2}(80: 20, v / v)$ [28]. The strain NIT-T3 was isolated from an anaerobic DS-AQDS agar plate which DS-basal medium supplemented with $10 \mathrm{mM}$ acetate, $5.0 \mathrm{mM}$ anthraquinone2,6-disulfonate (AQDS), $1 \mathrm{mM} \mathrm{Na}_{2} \mathrm{SO}_{4}$, and 1.5\% agarose. After 7-14 d of incubation at 
$28^{\circ} \mathrm{C}$, electrochemically active colonies showed orange halos, which was the color of the reduced form of AQDS. The colony culture was then purified via repeated agar-shake cultivation using DS-AF agar plate, DS-basal medium supplemented with $10 \mathrm{mM}$ acetate, $0.1 \%$ yeast extract, $1.5 \mathrm{mM} \mathrm{Na}_{2} \mathrm{~S}, 5.0 \mathrm{mM}$ fumarate, and $1.5 \%$ agarose. The purified culture was then phylogenetically identified based on the $16 \mathrm{~S}$ rRNA gene sequence amplified from the cell lysate [33] and named strain NIT-T3. Strain NIT-T3 was routinely cultured using the liquid DS-AF medium. In total, 7-14 d of anaerobic cultivation at $28^{\circ} \mathrm{C}$ was sufficient to achieve full growth.

\subsection{Morphological, Physiological, and Biochemical Analyses}

The morphology of strain NIT-T3 was evaluated using field-emission scanning electron microscopy (JSM-7800F; JEOL Ltd., Tokyo, Japan) operating at $1.0 \mathrm{kV}$; spore-forming ability and Gram staining nature were determined via optical microscopy, as described previously [34]. Motility was determined using the hanging drop method [34]. The effect of $\mathrm{NaCl}$ on cell activity was evaluated by monitoring growth in DS-AF medium supplemented with $0-8.0 \%(w / v) \mathrm{NaCl}$. The effect of $\mathrm{pH}$ was also determined using a bicarbonate-free medium adjusted to a $\mathrm{pH}$ ranging from 4.8 to 8.4 using sodium bicarbonate and by adjusting the $\mathrm{CO}_{2}$ concentration in the headspace gas. Temperatures ranging from $4{ }^{\circ} \mathrm{C}$ to $40{ }^{\circ} \mathrm{C}$ with approximate intervals of $5^{\circ} \mathrm{C}$ were applied to determine the effect on cell growth.

Formate, acetate, butyrate, lactate, pyruvate, succinate, malate, isopropanol, glucose, glycerol, isobutyrate, caproate, benzoate, phenol, methanol, ethanol, butanol, and fructose at $10 \mathrm{mM}$, and $0.5 \mathrm{~g} / \mathrm{L}$ peptone and yeast extract were tested as potential electron donors during nitrate reduction. Potential electron acceptors were evaluated by observing growth and detecting the oxidation of $5.0 \mathrm{mM}$ acetate in the presence of $10 \mathrm{mM}$ fumarate, $5 \mathrm{mM}$ nitrate, $10 \mathrm{mM}$ sulfate, $10 \mathrm{mM}$ thiosulfate, $5.0 \mathrm{mM}$ AQDS, and $10 \mathrm{mM}$ malate. Production of electric current by the strain NIT-T3 was evaluated via electrochemical cultivation using a graphite plate inoculated with NIT-T3, as described previously [35].

\subsection{Chemotaxonomic Analysis}

The cellular fatty acid composition and isoprenoid quinones present in NIT-T3 were investigated by Techno Suruga Laboratory Co., Ltd. (Shizuoka, Japan). Isoprenoid quinones were extracted, as described by Tamaoka et al. [36]. Cellular fatty acids were analyzed using cells cultured in liquid DS-AF medium at $28^{\circ} \mathrm{C}$ for $14 \mathrm{~d}$. The fatty acid profile was analyzed using the Sherlock Microbial Identification System version 6.0 (MIDI) using the TSBA6 database.

\subsection{Genetic Characterization}

Genomic DNA was extracted from strain NIT-T3, as described previously [37]. Sequencing was performed using a combination of the Illumina Miseq and Nanopore MinION. In total, $1.8 \mathrm{M}$ reads $(1.02 \mathrm{Gbp})$ of Illumina paired-end reads $(150 \times 2)$ and $0.37 \mathrm{M}$ Nanopore reads $(1.76 \mathrm{Gbp})$ were subjected to error removal using Short Read Manager and assembled using Unicycler-0.4.7. The complete genome of NIT-T3 was successfully determined, and gene prediction and genome annotation were performed using DFAST [38]. Comparison of the genes between NIT-T3 and other Desulfuromonas species was based on bidirectional best hits at $40 \%$ identity and $80 \%$ query coverage using SEED Viewer version 2.0 [39] and basic local alignment search tool in the NCBI database. The sequence of the NIT-T3 genome was deposited in the DNA Databank of Japan/GenBank under the accession number AP024355.

The $16 \mathrm{~S}$ rRNA gene sequences from all publicly available Desulfuromonas, Desulfuromusa, Geobacter, Geopsychrobacter, and Pelobacter genomes were downloaded from NCBI. A phylogenetic tree based on $16 \mathrm{~S}$ rRNA gene sequences of NIT-T3 and other members of the family Desulfuromonadaceae was generated using MEGA X based on the neighborjoining method [40]. 


\section{Results}

\subsection{Isolation of NIT-T3}

Anaerobic cultivation of AQDS-supplemented agar plates inoculated with the TGOA enrichment culture resulted in the formation of colonies that showed a change in color from colorless to orange. This suggested the ability of the colony to reduce AQDS and utilize it as an extracellular electron acceptor. A single colony was picked from the agar culture, purified via repeated agar-shake cultivation, and then re-cultivated in liquid DS-SF medium. Finally, based on the uniformity in microscopic morphology and 16S rRNA gene sequences, a liquid culture was selected and further purified by repeating the agar cultivation step. Cells of strain NIT-T3 were Gram-negative, non-spore-forming, rodshaped, and approximately $0.5 \mu \mathrm{m}$ in width and $1.5 \mu \mathrm{m}$ in length (Figure 1A).
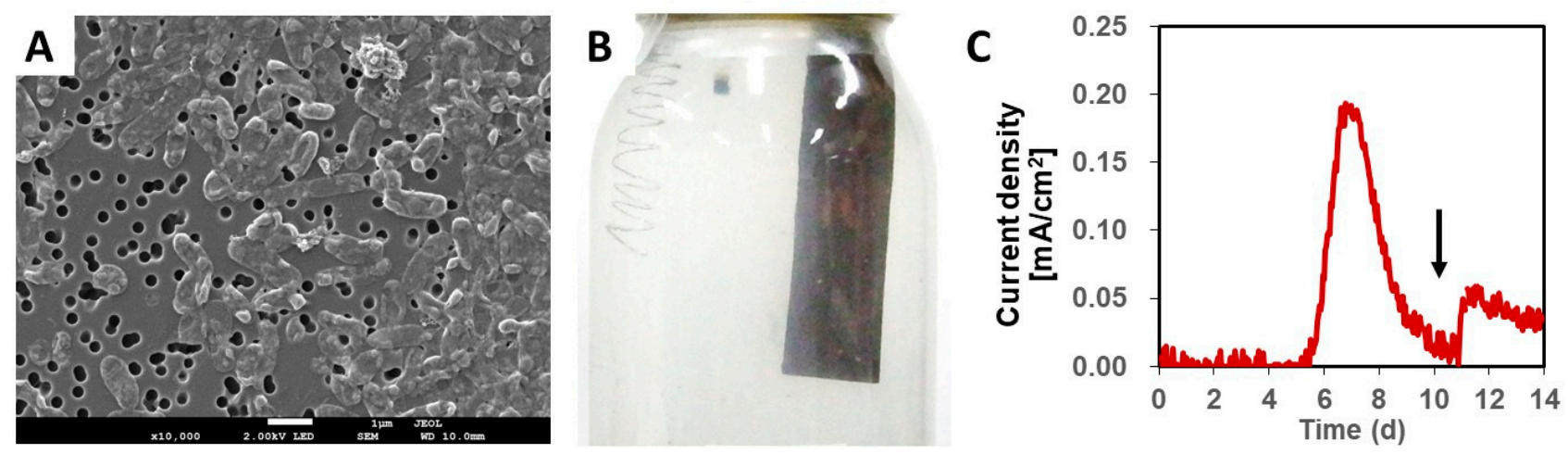

Figure 1. Morphology and electrogenic properties of strain NIT-T3. (A) Scanning electron microscopic image of strain NIT-T3. The white bar indicates $1 \mu \mathrm{m}$ of length. (B) Growth of a biofilm on an electrode in an electrochemical culture. (C) Electric current production by strain NIT-T3. The arrow in panel C indicates a spike of acetate addition.

The strain NIT-T3 produced an electric current on a graphite electrode in the presence of acetate and simultaneously generated a thin biofilm on the electrode surface (Figure 1B). The current in the electrochemical cultivation medium was rapidly generated and the maximum level was detected in the range of 0.18 to $0.19 \mathrm{~mA} / \mathrm{cm}^{2}$ on days 6 and 7 (Figure 1C). Electric current production decreased gradually with time; however, it increased immediately after adding acetate to the media. These results indicated that strain NIT-T3 grows by coupling EET to electrode with acetate-oxidation.

\subsection{Phylogenetic Identification Based on $16 S$ rRNA Sequencing}

Strain NIT-T3 was found to contain four 16S rRNA operons (rrn1-4); one of them showed $95.5-95.8 \%$ similarity to the three Desulfuromonas strains. The copy number was higher than that in Desulfuromonas strains containing two copies, and equal to that in four Geobacter and two Pelobacter strains: G. bemidjiensis, G. bremensis, P. acetylenicus, and P. propionicus. The $16 \mathrm{~S}$ rRNA gene-based phylogenetic tree revealed that all four rrns formed a cluster with sequences of five strains of the genus Desulfuromonas of the family Desulfuromonadaceae (Figure 2).

Strain NIT-T3 showed only $95.7 \%$ closest similarity based on $16 \mathrm{~S}$ rRNA gene sequences to that of D. michiganensis BB1 ${ }^{\mathrm{T}}$. Similarities with seven other species of the genus Desulfuromonas ranged from $92.9 \%$ to $95.4 \%$. The $16 \mathrm{~S}$ rRNA gene sequence similarity of strain NIT-T3 to members of the genera Pelobacter, Desulfuromusa, and Geobacter were 89.7-94.9\%, $90.7-90.9 \%$, and $90.6-91.6 \%$, respectively. According to the cut-off values of $98.2-99.0 \%$ [41], and $98.65 \%$ similarity among single species [42], the strain NIT-T3 may be proposed as a strain of a novel species of the genus Desulfuromonas. 


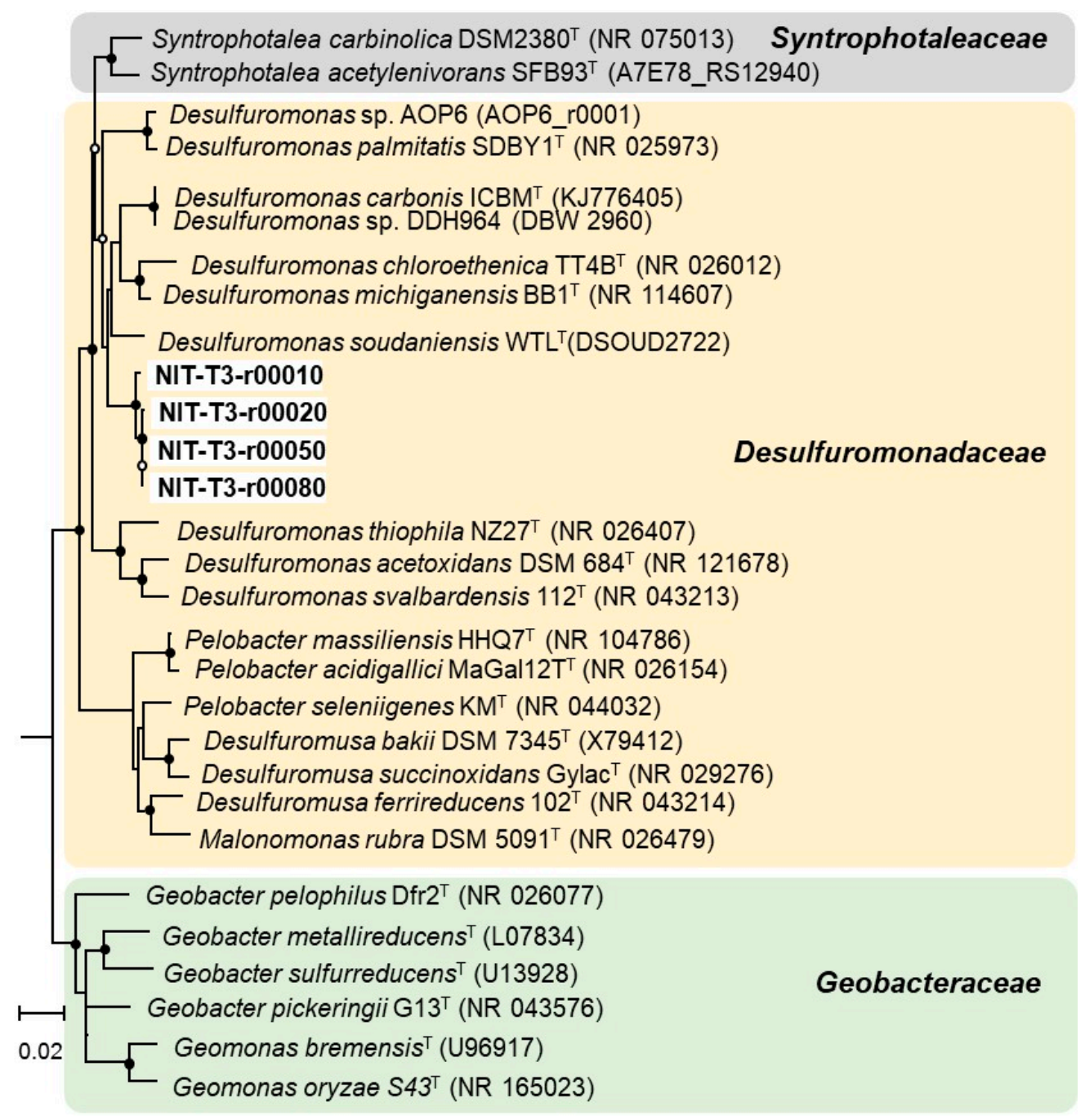

Figure 2. Phylogenetic tree generated using $16 \mathrm{~S}$ rRNA gene sequences of members of Desulfuromonadales. Closed and open circles indicate bootstrap $>80 \%$ and $60 \%$, respectively. GenBank accession numbers are stated in parentheses.

Strain NIT-T3 grew at $10-35^{\circ} \mathrm{C}$ (optimum, $25^{\circ} \mathrm{C}$ ), pH 6.4-8.4 (optimum pH 6.8-7.1), and tolerated $0.05-3 \% \mathrm{NaCl}$ (optimum, $0.2-1 \%$ ) (Table 1). Growth was completely inhibited at concentrations of $\geq 3 \% \mathrm{NaCl}$. Strain NIT-T3 could metabolize hydrogen, formate, acetate, lactate, pyruvate, succinate, malate, peptone, isopropanol, and yeast extract in the presence of nitrate (Table 1). Lactate, pyruvate, succinate, and the combination of $\mathrm{H}_{2}$ and acetate resulted in optimal growth together with nitrate. No growth occurred in the presence of butyrate, glucose, glycerol, ethanol, isobutyrate, caproate, benzoate, phenol, methanol, butanol, or fructose. Cells did not show any apparent movement on a slide, suggesting that the strain NIT-T3 was non-motile. 
Table 1. Characteristics of strain NIT-T3 and other Desulfuromonas strains.

\begin{tabular}{|c|c|c|c|c|c|c|c|c|c|c|}
\hline Characteristic & 1 & 2 & 3 & 4 & 5 & 6 & 7 & 8 & 9 & 10 \\
\hline Motility & NM & ND & NM & Motile & Motile & NM & Motile & $\mathrm{NM}$ & Motile & Motile \\
\hline $\mathrm{G}+\mathrm{C}$ content $(\%)$ & 63.1 & 61.2 & 61.2 & 50.1 & ND & ND & 61.6 & 54.7 & 62.3 & 62.3 \\
\hline $\begin{array}{l}\text { Temp. range }\left({ }^{\circ} \mathrm{C}\right) \\
\text { (Optimum) }\end{array}$ & $10-35(25)$ & 24 & $\begin{array}{c}10-37 \\
(25-37)\end{array}$ & $\begin{array}{l}2-20 \\
(14)\end{array}$ & $\begin{array}{c}10-35 \\
(25)\end{array}$ & $21-31$ & $\begin{array}{c}10-39 \\
(26-30)\end{array}$ & 40 & $(30-35)$ & $(30-35)$ \\
\hline pH range (Optimum) & $\begin{array}{c}6.4-8.4 \\
(6.8-7.1) *\end{array}$ & 6.8 & $\begin{array}{c}6.5-8.0 \\
(7.0)\end{array}$ & $\begin{array}{c}6.5-7.5 \\
(7.3)\end{array}$ & $\begin{array}{c}6.8-8.0 \\
(7.0-7.5)\end{array}$ & $\begin{array}{c}6.5-7.4 \\
(7.4)\end{array}$ & $\begin{array}{c}6.5-8.2 \\
(6.9-7.9)\end{array}$ & ND & $\begin{array}{c}6.4-8.5 \\
(7.6-7.8)\end{array}$ & $\begin{array}{l}6.4-8.5 \\
(7.6-7.8)\end{array}$ \\
\hline \multicolumn{11}{|l|}{ Electron donors } \\
\hline Hydrogen & + & + & $(+)$ & - & - & - & - & $(+)$ & - & - \\
\hline Lactate & + & + & + & - & + & - & - & + & - & - \\
\hline Fumarate & + & - & + & - & + & - & + & + & - & - \\
\hline Succinate & + & - & + & - & + & - & + & + & - & - \\
\hline Malate & + & ND & + & - & + & - & - & ND & - & - \\
\hline Acetate & + & + & + & + & + & + & + & + & + & + \\
\hline Pyruvate & + & + & + & + & + & + & + & - & ND & - \\
\hline Glucose & - & - & - & - & - & ND & - & - & - & - \\
\hline Butyrate & - & - & - & - & - & ND & - & - & - & - \\
\hline Glycerol & - & - & ND & - & - & ND & - & ND & - & - \\
\hline Peptone & + & ND & ND & ND & ND & ND & - & ND & ND & - \\
\hline Isopropanol & + & ND & ND & ND & ND & ND & ND & ND & ND & ND \\
\hline Ethanol & - & + & ND & + & - & - & - & - & - & + \\
\hline Benzoate & - & - & - & ND & ND & - & - & - & - & - \\
\hline Phenol & - & ND & - & ND & ND & ND & ND & - & ND & ND \\
\hline Fructose & - & ND & - & - & - & ND & - & - & - & - \\
\hline Isobutyrate & - & ND & ND & ND & ND & ND & ND & ND & ND & ND \\
\hline Caproate & - & ND & ND & ND & ND & ND & - & ND & - & ND \\
\hline Butanol & - & ND & ND & + & ND & ND & - & ND & - & + \\
\hline Fermentation of & Fumarate & ND & Fumarate & ND & $\begin{array}{l}\text { Fumarate } \\
\text { malate }\end{array}$ & None & ND & ND & ND & ND \\
\hline \multicolumn{11}{|l|}{ Electron acceptors } \\
\hline Nitrate & + & ND & - & - & - & - & - & - & - & - \\
\hline Sulfur & + & ND & + & + & + & ND & + & + & + & + \\
\hline Sulfate & - & ND & - & - & - & - & - & - & - & - \\
\hline Thiosulfate & - & ND & - & - & - & - & - & - & - & - \\
\hline Ferric iron (Fe(III)) & + & ND & + & + & + & + & $(+)$ & + & ND & ND \\
\hline Malate & + & ND & - & - & + & - & - & - & + & + \\
\hline Fumarate & ND & ND & - & + & + & + & - & + & + & + \\
\hline AQDS & + & ND & - & ND & ND & ND & ND & ND & ND & ND \\
\hline$\hat{\mathrm{GO}}$ & + & ND & ND & ND & ND & ND & ND & ND & ND & ND \\
\hline
\end{tabular}


Table 1. Cont.

\begin{tabular}{|c|c|c|c|c|c|c|c|c|c|c|}
\hline Characteristic & 1 & 2 & 3 & 4 & 5 & 6 & 7 & 8 & 9 & 10 \\
\hline Major fatty acids ( $>10 \%)$ & $\begin{array}{c}\mathrm{C}_{16: 1 \omega 7 \mathrm{c}}(26.2 \%) \\
\mathrm{C}_{16: 0}(18.3 \%) \\
\mathrm{C}_{15: 0}(13.2)\end{array}$ & ND & $\begin{array}{c}\mathrm{C}_{16: 0}(39.3 \%) \\
\mathrm{C}_{16: 1 \omega 7 \mathrm{c}} \text { and /or } \\
\text { iso- } \mathrm{C}_{15: 0} 2-\mathrm{OH} \\
(36.6 \%)\end{array}$ & $\begin{array}{c}\mathrm{C}_{16: 0}(43 \%) \\
\mathrm{C}_{16: 1} \omega 7 \mathrm{wc}(35 \%) \\
\mathrm{C}_{15: 0}(10 \%)\end{array}$ & ND & ND & ND & ND & ND & ND \\
\hline $\begin{array}{l}\text { Major respiratory } \\
\text { quinones }\end{array}$ & $\begin{array}{l}\text { MK-8 }(93 \%) \\
\text { MK-9 (5.3\%) } \\
\text { MK-7 (1.9\%) }\end{array}$ & ND & ND & ND & ND & ND & ND & ND & ND & ND \\
\hline \multicolumn{11}{|c|}{ 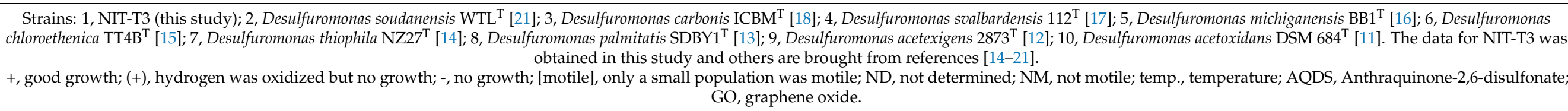 } \\
\hline
\end{tabular}

* Tested with acetate and fumarate as the substrates. 


\subsection{Physiological and Biochemical Characterization}

Similar to most Desulfuromonas species, strain NIT-T3 showed respiration using sulfur and ferric iron coupled with acetate oxidation. Contrary to the lack of nitrate respiration in other Desulfuromonas members, strain NIT-T3 exhibited nitrate respiration (Figure 3). The cell density increased from $5.7 \times 10^{7}$ to $2.4 \times 10^{8}$ cells $/ \mathrm{mL}$ with a reduction of $5.1 \mathrm{mM}$ of nitrate within $7 \mathrm{~d}$ of incubation. Meanwhile, a maximum concentration of $1.7 \mathrm{mM}$ of nitrite was produced in the medium. The NIT-T3 did not grow in the culture supplemented with nitrite. These results indicated the ability of strain NIT-T3 to grow on nitrate, while reducing nitrate to nitrite. The imbalance of the spiked nitrate and produced nitrite suggested the assimilative utilization of nitrite as nitrogen source.

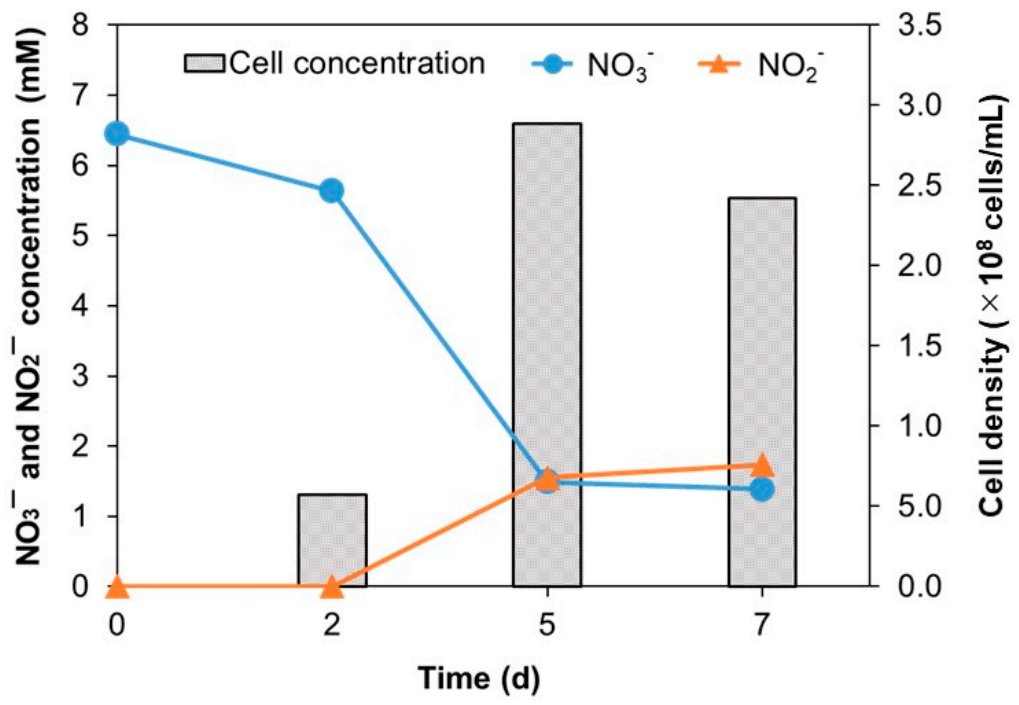

Figure 3. Changes in the $\mathrm{NO}_{3}{ }^{-}$and $\mathrm{NO}_{2}{ }^{-}$concentrations and NIT-T3 cell densities in the culture supplemented with nitrate.

\subsection{Chemotaxonomic Characterization}

The major fatty acids identified in strain NIT-T3 were $C_{16: 1} \omega 7 c(26 \%), C_{16: 0}(18 \%)$, and $C_{15: 0}(13 \%)$ (Table 1). The fatty acid profile of Desulfuromonas species is available for only two strains, D. svalbardensis $112^{\mathrm{T}}$ [20] and D. carbonis $\mathrm{ICBM}^{\mathrm{T}}$ [21]; these two strains contain the three major fatty acids found in strain NIT-T3. However, the proportions differ among the three strains. The most abundant fatty acid is $\mathrm{C}_{16: 0}$ (approximately $40 \%$ ) in strains $112^{\mathrm{T}}$ and $\mathrm{ICBM}^{\mathrm{T}}$, and $\mathrm{C}_{15: 0}$ in ICBMT is detected in minor amounts $(0.1 \%)$. The presence of $C_{16: 1} \omega 7 c$ and $C_{16: 0}$ as main cellular fatty acids was common in most members of Geobacter [43-47] rather than in only Desulfuromonas.

The major respiratory quinones in NIT-T3 were identified as MK-8 (93\%), and other menaquinones including MK-7 (1.9\%) and MK-9 (5.3\%) were detected. This result is consistent with the fact that MK-8 is a typical respiratory quinone present in the genus Geobacter $[43,44,47,48]$, although the menaquinone profile data of other species of the genus Desulfuromonas are not available.

\subsection{General Genomic Features}

NIT-T3 contained a single 4,656,376 bp circular chromosome that encodes 4119 proteincoding sequences (CDS), 60 transfer RNAs, 1 transfer-messenger RNA, and 10 rRNAs. The G + C content in strain NIT-T3 was approximately $63.1 \%$, which is similar to that of D. acetexigens $2873^{\mathrm{T}}$ and D. acetoxidans (Table 1). The genome map is shown in Figure 4. Regarding energy conversion, NIT-T3 had CDSs to metabolize hydrogen, lactate, pyruvate, and other organic acids of the TCA cycle intermediates (fumarate, succinate, malate), and complete TCA cycle. NIT-T3 also contained a full set of genes associated with glycolysis similar to the other two strains; however, the bacteria cannot utilize glucose due to the lack 
of glucose transporters [25]. A gene encoding nitrate reductase (NarB) was found which oxidizes quinol and reduces nitrate to nitrite. The reductive acetyl-CoA pathway and reductive TCA cycle lacked certain genes, suggesting an inability of the strain to fix carbon.

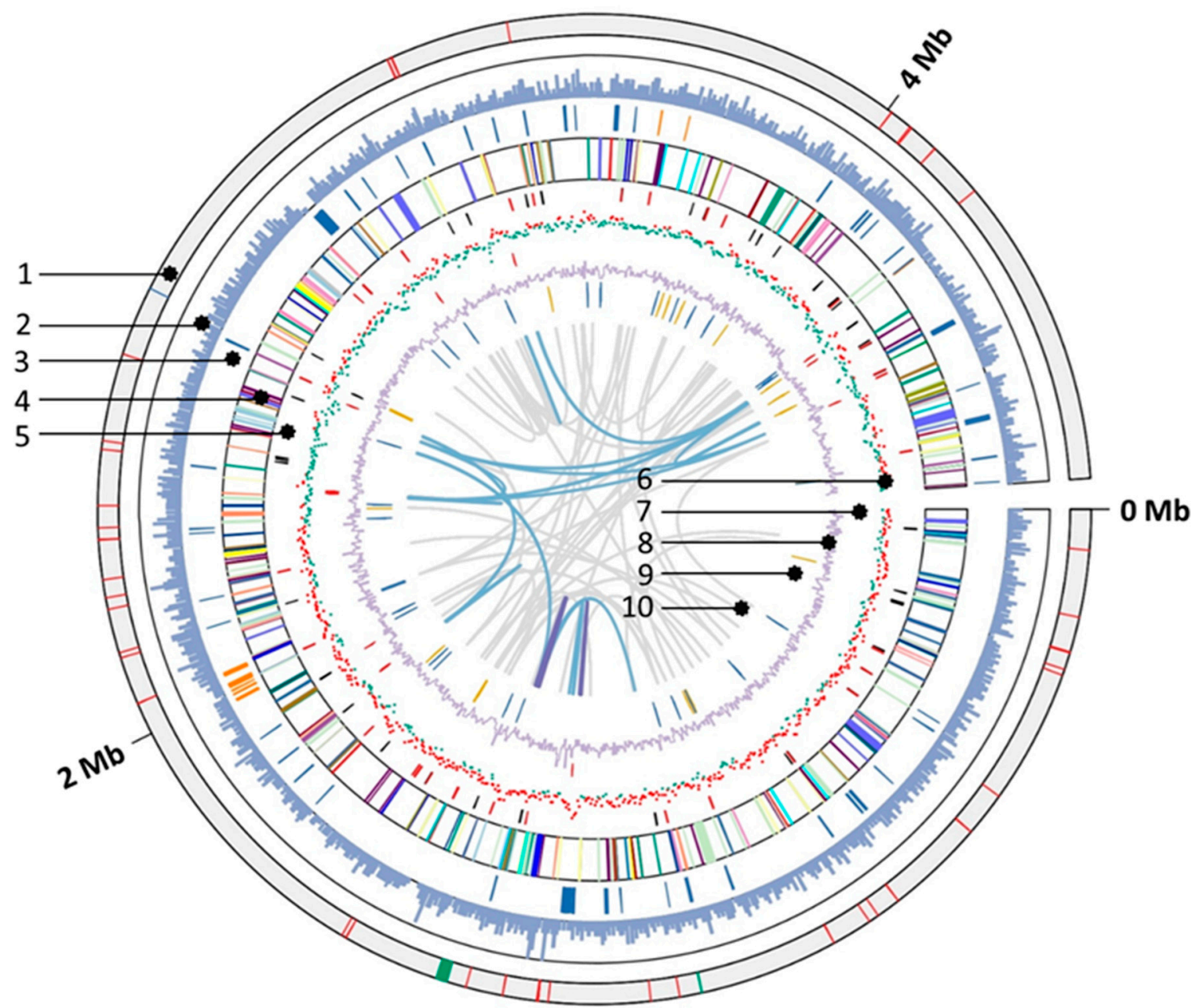

Figure 4. Features of the complete genome of 'Desulfuromonas versatilis' NIT-T3. Circular representation of the genome was generated using TBtools v.1.082 [49]. Rings numbered from the outside to inside are: 1, location of tRNA (red), transfer messenger RNA (blue), and rRNA (green); 2, gene density; 3, c-type cytochromes (blue), and type IV pili (orange); 4, protein coding sequences colored based on KEGG category; 5 , putative sensor histidine kinases (red) and response regulators (black); 6, G + C skew (red, positive; green, negative) approximated using GenSkew (http://genskew.csb.univie.ac.at); 7, transposase (red) and phage integrase (black); 8, G + C content; 9, genes unique to NIT-T3 (orange, annotated; blue, unannotated) compared to other Desulfuromonas spp; 10 , links showing repetitive sequence $\geq 95 \%$ identity (cyan, $>500 \mathrm{bp}$; purple, $>2 \mathrm{kbp}$ ). KEGG, Kyoto Encyclopedia of Genes and Genomes.

\subsection{Putative c-Type Cytochromes}

NIT-T3 possessed 79 putative $c$-type cytochromes. The CDSs with $\mathrm{C}(\mathrm{X})_{n} \mathrm{CH}, n=2-4$, were first screened and then identified as $c$-type cytochromes either based on the presence of a conserved domain of $c$-type cytochromes in the pfam and TIGR databases (e-value $=<0.01$ cutoff) or showing an e-value $<10^{-10}$ in the pairwise alignment with $c$-type cytochromes of strain PCA. Among them, 61 were homologs of $c$-type cytochromes in Desulfuromonas strains (Figure $5, \geq 40 \%$ amino acid identity (AAI) in the 759-92 AAs of the HIPER scoring region) and 38 were homologs of those in Geobacter sulfurreducens PCA, a well-characterized model of iron-reducing bacterium. NIT-T3 contained a relatively larger number of $c$-type cytochromes than other Desulfuromonas strains in the range from 37 to 44 . Based on PROSITE prediction [50], most of the $c$-type cytochromes were present 
in the periplasmic (28), extracellular (7), and cytoplasmic membranes (6), whereas a few cytochromes were present in the cytoplasm (3). The number of heme-binding motifs varied, and ranged from 1 to 53 .

\begin{tabular}{|c|c|c|c|c|c|c|c|c|c|c|c|}
\hline \multirow{4}{*}{ Tag } & \multirow{2}{*}{\multicolumn{3}{|c|}{ Number of }} & & & & & Identity (\%) & $20-3030$ & $-40 \quad 41-5 c$ & $50 \quad 51-60 \quad 61-70$ \\
\hline & & & & Crocost nxoto & in stroins & & & Closest protein in databe & ase & & \\
\hline & $\mathrm{CX}_{\mathrm{n}} \mathrm{C}$ & & Local- & Closest prote & In strains & & & & Phyloge & eny of or & origin \\
\hline & $\mathrm{n}=2$ & $n=3$ & ization & PCA1 & DDH964 & AOP6 & WTL & Accession & Family & Gen. & Species \\
\hline 3570 & 5 & 0 & CM & OmcV, 1996 & & & 2438 & FD174_3512 & Geo & & - \\
\hline 3730 & 4 & 0 & & PpcD, 1024 & 2382 & 1767 & 2694 & C0619 13365 & Des & Desl & - \\
\hline 4810 & 2 & 0 & CM & CcoP, 2513 & 2374 & 0638 & 2315 & C0615 07200 & Des & Desl & - \\
\hline 5430 & 53 & 0 & PP & OmcX, 0670 & 301 & 1132 & 2958 & WP_040198997 & Geo & Geoa & subterraneus \\
\hline 5440 & 12 & 0 & PP & ExtA, 2645 & - & 1132 & - & ENN94_00220 & Geo & Geoa & subterraneus \\
\hline 5490 & 10 & 0 & - & ExtG, 2724 & 1658 & & 823 & IH614_00830 & & 年 & - \\
\hline 6100 & 18 & 0 & PP & CytT, 2299 & 833 & 982 & 2629 & C0623_04030 & Des & Desl & 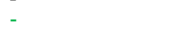 \\
\hline 7890 & 1 & 0 & PP & - & 839 & 2693 & 601 & DSOUD 0601 & Des & Desl & soudanensis \\
\hline 7900 & 3 & 0 & $\mathrm{CP}$ & - & - & - & - & BA871_12945 & & & - \\
\hline 8600 & 8 & 0 & EX & OmcZ, 2076 & - & - & - & GFER_11920 & Geo & Geoa & ferrihydriticus \\
\hline 8630 & 2 & 0 & - & Dhc2, 2927 & 3061 & 2447 & 649 & C0614_03360 & Des & Desl & - \\
\hline 9000 & 3 & 0 & PP & PpcD, 1024 & 1072 & 1210 & 3086 & IBX47_10010 & & & - \\
\hline 9780 & 6 & 0 & PP & 2211 & 2875 & 115 & 2475 & DBW 2875 & Des & Desl & - \\
\hline 9980 & 12 & 0 & PP & 615 & 2851 & 1175 & 2958 & FL622_12825 & Des & $\begin{array}{l}\text { Desl } \\
\text { Desl }\end{array}$ & acetexigens \\
\hline 10000 & 8 & 0 & PP & $\mathrm{CtcB}, 0616$ & 2849 & 1668 & 2956 & A2X84_08365 & Des & - & - \\
\hline 10020 & 4 & 0 & EX & OmcE, 0618 & 2847 & 1181 & 2954 & C0621_05920 & Des & Desl & - \\
\hline 10580 & 7 & 1 & - & 3218 & 2851 & 1175 & 2958 & C0622_11315 & Des & Desl & - \\
\hline 10590 & 12 & 0 & - & 670 & & 1173 & & HPP94_15845 & - & 1 & - \\
\hline 10600 & 10 & 2 & EX & 3226 & 3049 & 1181 & 663 & C0619 06915 & Des & Desl & - \\
\hline 10630 & 5 & 0 & [x & ExtT, 3223 & 2851 & 1178 & $\begin{array}{l}003 \\
2958\end{array}$ & GSVR 15810 & Geo & Geob & - \\
\hline 10650 & 13 & 1 & PP & 3218 & 2851 & 1175 & 2958 & ENR76_08625 & Geo & - & - \\
\hline 10660 & 10 & 0 & EX & OmcB, 2737 & 2625 & 2385 & & GM21 3565 & Geo & Geob & - \\
\hline 10670 & 18 & 0 & $\mathrm{PP}$ & OmcB, 2737 & 3049 & 2432 & 2970 & GPEL0_01f5367 & Geo & Geob & pelophilus \\
\hline 10680 & 4 & 0 & PP & CtcA, 3221 & 2847 & 1176 & 2954 & GSVR_15740 & Geo & Geob & - \\
\hline 10700 & 22 & 0 & EX & OmcB, 2737 & 2625 & 2432 & & C0619_07865 & Des & Desl & - \\
\hline 10710 & 4 & 0 & $\mathrm{PP}$ & CtcA, 3221 & 2847 & 1176 & 2954 & GSVR 15740 & Geo & Geob & - \\
\hline 12140 & 5 & 0 & - & CbcX, 1648 & 2721 & 764 & 2440 & C0617_08690 & Des & Desl & - \\
\hline 15020 & 2 & 0 & - & - & - & - & - & - & - & ( & - \\
\hline 15500 & 2 & 0 & PP & 1538 & - & 104 & 3080 & DCZ63_04680 & Geo & Geob & - \\
\hline 15840 & 8 & 0 & PP & $=$ & 1324 & - & 1989 & GQ530_06150 & - & - & - \\
\hline 16340 & $\stackrel{\circ}{1}$ & 0 & - & - & 3541 & - & 3220 & SAMN05660420_00810 & Des & Desl & kysingii \\
\hline 18560 & 3 & 0 & PP & PpcD, 1024 & 1072 & 1210 & 3086 & JXR59 05200 & Des & - & - \\
\hline 19090 & 1 & 0 & & СусC, 1740 & 1735 & - & - & C0623_12135 & Des & Desl & - \\
\hline 20270 & $\frac{1}{7}$ & 0 & PP & 3214 & $1 / 35$ & 5 & - & B5V00 09970 & Geo & Geot & hydrogeniphilus \\
\hline 20370 & 9 & 1 & PP & Omcl, 1228 & 1987 & 1517 & 3216 & AOP6 1517 & Des & Desl & - \\
\hline 21530 & 7 & 0 & PP & & & 87 & 1849 & DSOUD_1849 & Des & Desl & soudanensis \\
\hline 23710 & 4 & 0 & - & 3154 & - & 1033 & 150 & DSOUD_0150 & Des & Desl & soudanensis \\
\hline 23720 & 4 & 0 & & $\mathrm{NrfH}, 3155$ & - & 1033 & 151 & GeB 11375 & Geo & Geoa & subterraneus \\
\hline 26390 & 4 & 0 & EX & OmcP, 2913 & 3049 & 1181 & 663 & - & Geo & Geop & electrodiphilus \\
\hline 26400 & 35 & 2 & - & 2884 & 3048 & -101 & 664 & - & Geo & Geoa & subterraneus \\
\hline 26410 & 15 & $\overline{1}$ & - & OmcN, 2898 & 3039 & - & 665 & F0488_05880 & Geo & Geob & - \\
\hline 26420 & 21 & 1 & EX & OmcN, 2898 & 3047 & - & 665 & DBW_3047 & Des & Desl & - \\
\hline 26940 & 12 & 0 & $\overrightarrow{\mathrm{PP}}$ & ObcM, 2935 & - & 659 & 00 & C0617_13980 & Des & Desl & - \\
\hline 27260 & 1 & 0 & & & & & 2537 & & & Dor & - \\
\hline 27920 & 4 & 0 & PP & PpcD, 1024 & 2382 & 1063 & 2694 & DSOUD_2694 & Des & Desl & soudanensis \\
\hline 28400 & 7 & 1 & $\mathrm{CP}$ & 1786 & 2445 & 1182 & 2414 & A2X84_02795 & & & \\
\hline 28410 & 4 & 0 & - & 1787 & 2446 & 1181 & 2415 & - & Geo & Geop & electrodiphilus \\
\hline 29000 & 2 & 0 & - & & & & & GW001_04425 & 400 & - & - \\
\hline 29420 & 6 & 0 & PP & CytT, 2299 & 833 & 2967 & 2629 & ENQ87_05415 & Geo & Geob & metallireducens \\
\hline 29940 & 12 & 0 & $\mathrm{PP}$ & ExtA, 2645 & -835 & 1133 & $20 \angle 9$ & C0616 11920 & $\begin{array}{l}\text { Heo } \\
\text { Des }\end{array}$ & Desl & metamireducens \\
\hline 29950 & 12 & 0 & $\mathrm{PP}$ & OmcX, 0670 & - & 1132 & - & WP_140396673 & Des & - & - \\
\hline 30630 & 13 & 0 & - & OmcB, 2737 & 3210 & 456 & - & - & Des & Desl & - \\
\hline 30640 & 10 & 0 & PP & OmaB, 2738 & 3211 & 457 & - & - & Des & Desl & - \\
\hline 30830 & 4 & 0 & - & & - & - & 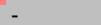 & - & - & - & - \\
\hline 31280 & 2 & 0 & CM & CoxB, 0222 & 3011 & 0614 & RS04825 & WP 148895441 & Geo & Geot & ehrlichii \\
\hline 31490 & 4 & 0 & - & CbcR, 2930 & & 654 & Wu & IH614 13215 & - & - & - \\
\hline 31530 & 10 & 0 & - & CbcM, 2934 & - & 658 & - & C0617_16150 & Des & Desl & - \\
\hline 31540 & 12 & 0 & - & CbcM, 2935 & 3210 & 659 & 2970 & C0618_06315 & Des & Desl & - \\
\hline 31560 & 5 & 0 & - & ExtK, 2937 & & 662 & - & IH613_02405 & - & - & - \\
\hline 31880 & 1 & 0 & $\mathrm{CM}$ & Cyc1, 3334 & 948 & 615 & 963 & C0617_01390 & Des & Desl & - \\
\hline 35490 & $\frac{1}{5}$ & 0 & $\mathrm{CP}$ & CtcC, 2801 & -940 & 276 & 90 & HGB35_03625 & Geo & - & - \\
\hline 36250 & 9 & 0 & - & ExtD, 2642 & 2791 & 2 & 704 & ENR76 02965 & Geo & - & - \\
\hline 36260 & 5 & 0 & - & ExtCF, 2643 & 2792 & - & 2914 & 04185 & - & & \\
\hline 36350 & 1 & 0 & - & 1397 & 213 & - & 291 & IH614_16780 & $=$ & - & - \\
\hline 36530 & 1 & 0 & - & PpcC, & 2382 & 1768 & 2694 & IH614_03740 & - & - & - \\
\hline 37260 & 7 & 0 & - & $\mathrm{CbcA}$ & & 2567 & 1253 & & Des & Desl & acetexigens \\
\hline 37510 & 3 & 0 & - & OmcV, 1996 & 2875 & 2520 & 2475 & FL622_16595 & Des & Desl & acetexigens \\
\hline 38420 & 10 & 0 & PP & OmaB, 2738 & 3211 & 457 & & DBW 262 & Des & Desl. & - \\
\hline 38430 & 12 & 0 & EX & OmcB & 262 & 2432 & & C0614_09110 & Des & Desl & \\
\hline 38440 & 10 & 0 & $\mathrm{PP}$ & OmaB, 2738 & 3211 & 456 & - & DB & Des & Desl & - \\
\hline 38450 & 10 & 0 & - & OmcB, 2737 & 0.3379 & 2432 & 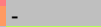 & GSU2737 & Geo & Geob & sulfurreducens \\
\hline 38460 & 10 & 0 & - & - & 3209 & & 887 & C0617 16065 & Des & Desl & - \\
\hline 39480 & 1 & 0 & - & 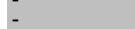 & 3572 & 2837 & 81 & FIB02_09325 & Des & Desl & 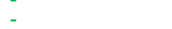 \\
\hline 40040 & 9 & 0 & CM & $\mathrm{Cbc}$ & 421 & 128 & 214 & A2X84_12435 & Des & - & - \\
\hline 60 & 4 & 0 & - & & 41 & - & 21 & A2 & Des & - & - \\
\hline 40090 & $\begin{array}{l}4 \\
5\end{array}$ & 1 & $\mathrm{CM}$ & ImcH, 3259 & 412 & 11 & 207 & DBW_0412 & Des & Desl & 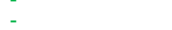 \\
\hline 40120 & 2 & 0 & PP & MacA, 0466 & 1968 & 558 & RS12400 & WP 092058305 & Des & Desl & acetexigens \\
\hline 40720 & 4 & 0 & $\mathrm{PP}$ & NrfA, 3154 & - & 1033 & 150 & TR077878 & Des & Desl & acetoxidans \\
\hline 41160 & 2 & 0 & & 3332 & 13 & 12 & 3572 & PLX84049 & Des & Desl & - \\
\hline
\end{tabular}

Figure 5. List of putative $c$-type cytochrome $C$ proteins present in the NIT-T3 genome. Tag indicates the locus tag of the coding sequence encoding $c$-type cytochromes in the genome. CM, cytoplasmic membrane; PP, periplasm; EX, extracellular; (-), unknown. The number and color scale for the closest protein in the strain indicates the locus tag and amino acid identity (\%), respectively. Geo, Geobacteraceae; Geoa, Geoalkalibacter; Geob, Geobacter; Geop; Geopsychrobacter; Geot, Geothermobacter; Syn, Syntrophotaleaceae; Synt, Syntrophotalea; Des, Desulfuromonadaceae; Desl, Desulfuromonas.

The $c$-type cytochromes were highly variable showing approximately $\sim 77.76 \%$ similarity to those of Desulfuromonas strains. The phylogeny matched within families of Geobacteraceae and Desulfuromonadaceae, and few were related to cytochromes found only 
in metagenomes. Among them, six c-type cytochromes were unique and shared $<40 \%$ AAI with those in the database; four independent genes (DESUT3_0860, 20270, 29420, DESUT3_29000), and two (DESUT3_10660, 38460) were present in gene clusters of putative cytochromes (Figure 6).

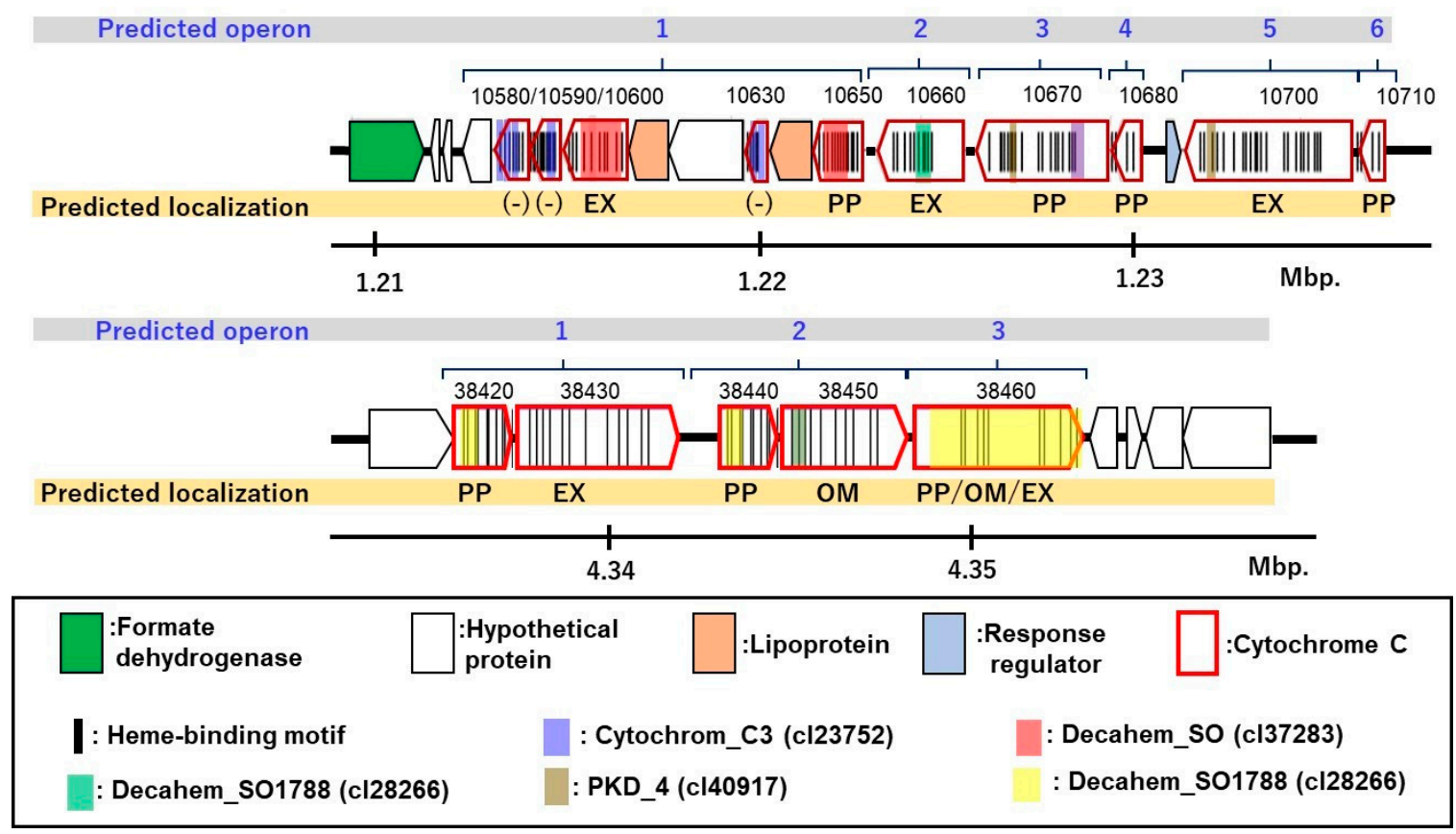

Figure 6. Two predicted gene clusters of unique $c$-type cytochrome $C$ in strain NIT-T3.

\subsection{Homologs of c-Type Cytochromes to Those in G. sulfurreducens PCA}

Twenty-four c-type cytochromes were homologs of those functionally identified in G. sulfurreducens PCA. DESUT3_09000, 18560, and 27920 are homologous to PpcD, DESUT3_10670 and 38450 are homologous to OmcB, and DESUT3_40090 and 40040 are homologous to ImcH and CbcL present in G. sulfurreducens, respectively. These cytochromes are involved in the porin-cytochrome (PcC) EET pathways that mediate electron transfer across the cell envelope [51]. In the Pcc pathways, ImcH and $\mathrm{CbcL}$ represent inner membrane cytochromes that oxidize quinol in the cytoplasmic membrane and transfer the released electrons to the periplasmic PpcA/PpcD [52,53]; PpcA/PpcD transfers the electrons acquired from the cytoplasm to the OmcB-based (ombB-omaB-omcB) conduit in the outer membrane [54]. The OmcB-based conduit transfers electrons through the lipid bilayer of proteoliposomes and directly reduces Fe(III) hydroxides outside the proteoliposomes [55,56].

DESUT3_29940, 36260, 05490, 10630, and 31560 are homologs of ExtA, ExtC, ExtG, ExtT, and ExtK of G. sulfurreducens, respectively. They belong to the outer membrane electron conduit ext gene clusters, which transfer electrons across the outer membrane to the bacterial surface [57]. DESUT3_31530 and 31540 are homologs of CbcM, and DESUT3_37260, 31490, 40060, and 12140 are homologs of CbcA, CbcR, CbcS, and CbcX of G. sulfurreducens, respectively. These $\mathrm{Cbc}$-proteins and $\mathrm{CbcL}$ constitute the menaquinol oxidoreductase protein complexes, which combine electron transfer processes to form a proton gradient across the inner membrane via either a $Q$ loop or a $Q$ cycle $[58,59]$.

DESUT3_40120 is a homolog of the inner-membrane-associated diheme cytochrome MacA of G. sulfurreducens, which is described as a peroxidase that can also mediate the electron transfer between inner membrane components and multiheme periplasmic cytochromes [60]. DESUT3_20370, 37510, and 29950 are homologs of the outer membrane cytochrome OmcI, OmcV, and OmcX of G. sulfurreducens, respectively. These Omc-proteins help transfer the electrons extracellularly, are required for Fe (III) reduction [61,62], and 
are significantly upregulated by $\mathrm{Fe}(\mathrm{III})$ oxides/Mn(IV) oxides/granular activated carbon $[61,63]$. DESUT3_40720 is a homolog of NrfA, which functions as a nitrite reductase component in a putative $\mathrm{NrfH} / \mathrm{NrfA}$ nitrite-to-ammonia respiration pathway [25]. DESUT3_31280 is a homolog of CoxB encoding a cytochrome c oxidase subunit II, which is involved in the oxidative phosphorylation pathway.

\subsection{Type IV Pilus (T4P)-Related Genes}

The strain NIT-T3 was found to contain 16 CDSs encoding T4P (Figure 7) and 3 related genes including transcription regulators. T4P are filamentous polymers of pilin monomers that undergo dynamic rapid polymerization and depolymerization from a pool of pilin [64]. The pilus polymer of pilin protein PilA of G. sulfurreducens PCA is an electrically conductive pilus and is known to be involved in the EET of solid electron acceptors [65]. Aromatic acids are key elements associated with conductivity and are estimated to account for $9.83 \%$ of the content in the PilA of strain PCA [66]. The aromatic acid content in the PilA homolog in NIT-T3 was $14.2 \%$, which was relatively high based on the range of PilA aromatic acid content in phylogenetically diverse bacteria (5.5-25.25\%) [67]; this suggested that the polymer may be conductive. The NIT-T3 genome was found to contain a full set of genes encoding T4P: two major pilins PilA and PilE, four minor pilins PilE, PilY, PilV, and PilW, and other essential proteins for the secretin (PilQ), alignment (PilM, PilN, PilO, and PilP), platform (PilC), four retraction ATPases (PilT), and assembly ATPases (PilB). Most CDSs are well-conserved in the genus Desulfuromonas, except for CDSs of four minor pilins closely related to the genera Syntrophotalea (PilE, PilV, and PilW) and Geobacter (PilY).

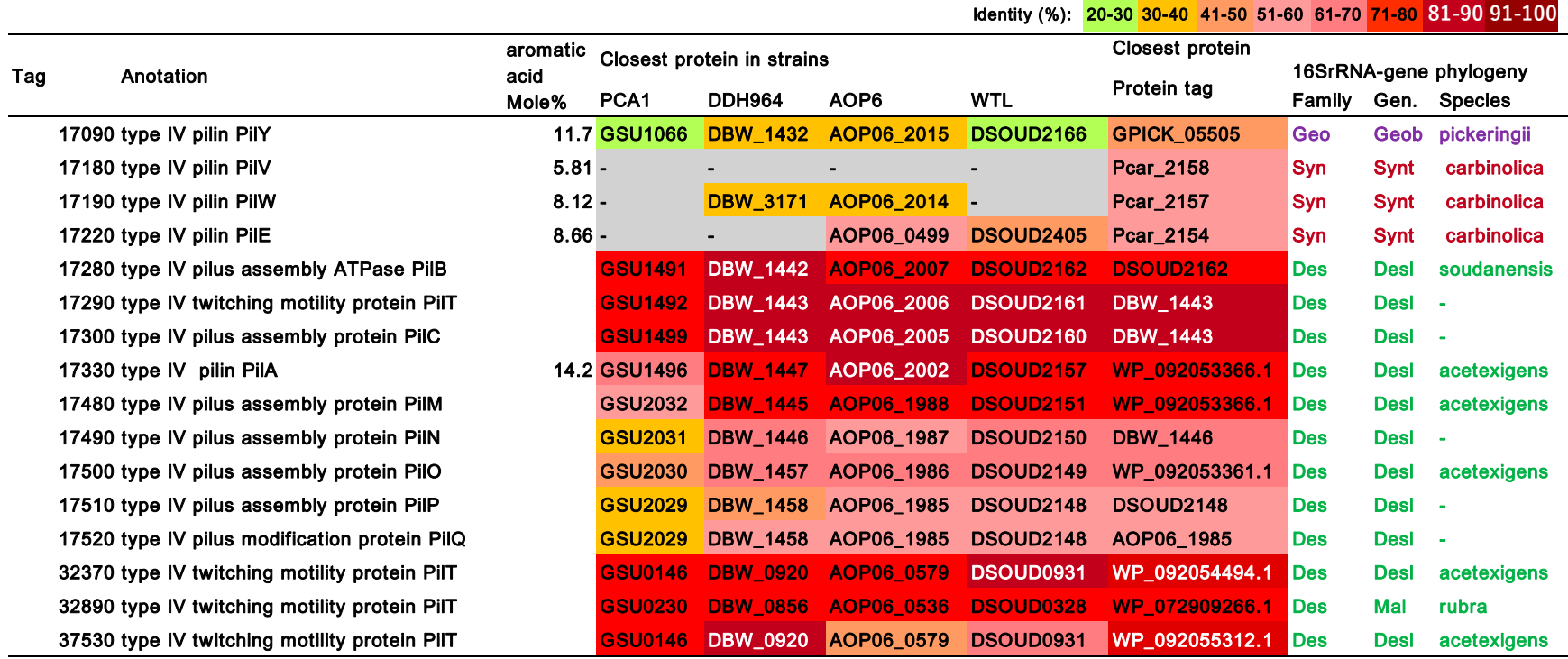

Figure 7. List of T4P assembly-related genes in the NIT-T3 genome Tag indicates the locus tag of coding sequences encoding T4P-related proteins in the genome. The number and color scale for the closest protein in the strain indicates the locus tag and amino acid identity (\%), respectively. T4P, Type IV pilli; Geo, Geobacteraceae; Geob, Geobacter; Syn, Syntrophotaleaceae; Synt, Syntrophotalea; Des, Desulfuromonadaceae; Desl, Desulfuromonas; Mal, Malonomonas.

\section{Discussion}

The isolation of a novel electrogenic strain NIT-T3 of the genus Desulfuromonas and its respiration-ability specific to solid minerals suggests the adaptation of the genus to solid minerals or conductor-driven metabolism [8-13]. The ability to grow on solid minerals and electrodes is supported by the presence of seven extracellular c-type cytochromes (Figure 5), and conductive pilin (PilA)-homologs and full sets of T4P-assembly genes in the genome (Figure 6). These CDSs are phylogenetically cross-related in the order Desulfuromonadales beyond family, and show higher identities with CDSs of the genera Desulfuromonas and Malonomonas of the family Desulfuromonadaceae; Geobacter, Geoalkalibacter, and Geopsychrobacter 
of the family Geobacteraceae, and Syntrophotalea of family Syntrophotaleaceae. The fact that all members in these genera are capable of reducing solid minerals, and that the EET-related genes are closely related suggests the early divergence of EET during the evolution of bacteria in the order Desulfuromonadales. The strain NIT-T3 required $\mathrm{NaCl}$ and showed growth in the presence of $0.05-3.0 \% \mathrm{NaCl}$; the preference for $\mathrm{NaCl}$ was in agreement with the salt tolerance observed in the genus Desulfuromonas (0-3.0\%) [17,19,21]. This supports the fact that Desulfuromonas species have widely adapted to both marine and freshwater environments.

The $c$-type cytochromes were hypervariable and abundant $(n=79)$, whereas other Desulfuromonas strains are reported to contain 37-44 c-type cytochromes according to the given annotation. The difference in the number was probably due to the difference in annotation strategies. A CDS for c-type cytochrome (DESUT3_5430) had 53 heme-binding sites which was the maximum number, and showed $54 \%$ of the closest identity to that of Geoalkalibacter subterraneus (B_0221) and 49\% and 41\% with that of contigs of D. acetexigens and M. rubra, respectively. The number of heme-binding sites in these homologs ranged from 55 to 77, indicating the broad distribution of the $c$-type cytochrome with such a large number of heme-binding sites in the order Desulfuromonadales.

Among the unique c-type cytochromes, two cytochromes (DESUT3_10660 and DESUT3_38460) were present in gene clusters including multiple c-type cytochromes (Figure 7). The gene cluster harboring DESUT3_10660 contains ten non-cytoplasmic c-type cytochromes with domains for cytochrome_C3 (cl23752), decahem_SO (cl37283), and decahem_SO1788 (cl28266). Three homologs of $c$-type cytochromes are found in strain PCA: DESUT3_10630 sharing 49\% identity with ExtT which is a subunit of ExtTUVW conduit, and DESUT3_10680 and DESUT3_10710 with both sharing 43\% identity with CtcA and tetra/tri-heme $c$-type cytochromes, respectively. DESUT3_38460 was located in a gene cluster including five CDSs of $c$-type cytochromes: three CDSs (DESUT3_38420, 38440, and 38450) shared 41-69\% similarity with the c-type cytochrome of Desulfuromonas strains, and DESUT3_38430 showed 53\% similarity with a hypothetical protein of Desulfuromusa kysingii. Among them, only one CDS (DESUT3_38450) was the homolog of the functionally identified $c$-type cytochrome known as omcB, a subunit of the OmaB/OmbB/OmcB conduit. The function of these gene clusters is unknown; however, it is speculated that they are essential for the unique adaptation of the strain for facilitating EET.

Strain NIT-T3 could metabolize nitrate which is not observed in other strains in the genus Desulfuromonas. The strain NIT-T3 produced nitrite; however, its production was approximately one-third of the amount of nitrate supplemented (Figure 3). The difference in the concentrations of produced nitrite and supplemented nitrate may be attributed to the assimilation of ammonia produced from nitrite. Analysis of the genome of NIT-T3 indicated the possibility of nitrite-to-ammonia transformation. Similar to NIT-T3, the complete genomes of all four Desulfuromonas strains contain CDSs for the nitrogen fixation pathway, including nitrite-to-ammonia reduction. However, genes encoding transporters for the uptake of extracellular nitrate were present in NIT-T3 alone (Figure 8A). This is consistent with the fact that NIT-T3 alone, and not the other Desulfuromonas strains, could reduce nitrate.

The NIT-T3 genome was found to contain several unique CDSs that encode complete metabolic pathways which are absent in the other three Desulfuromonas genomes: histidine degradation to glutamate (Figure 8B), and C1-unit interconversion (Figure 8C). This suggested a variable metabolism in members of the genus Desulfuromonas associated with the transient reduction and uptake of genes in the genome.

In addition to the unique genes mentioned above, NIT-T3 possesses 46 other CDSs in the genome with no confirmed homologs $(<40 \%)$ in other species of the Desulfuromonas strains; 29 of these sequences encode proteins with predicted functional annotation (Table S1). The unique proteins include a putative autoinducer-2 (AI-2) transporter family protein (DESUT3_07100), which has been reported to regulate the intracellular concentration of AI-2, a quorum-sensing chemical that affects global gene expression in biofilms $[68,69]$ and 
shares a higher identity with proteins of Geobacter species than those of Desulfuromonas. The NIT-T3 genome includes another candidate for the AI-2 transporter family protein (DESUT3_31000) that was similar to that in all three genomes of the genus Desulfuromonas, but not to that of Geobacter species (63-70\% identity). These two paralogs of AI-2 transporters with different phylogenies may potentially control biofilm growth. DESUT3_07210 encodes RNA molecules of a labile antitoxin, HicB, that regulates the expression of a toxin protein, HicA. Both proteins are involved in bacterial survival under stress conditions, such as nutrient deprivation and antibiotics $[68,69]$. An additional set of CDSs for HicAB was present in the genome; however, its role in Desulfuromonadales has never been investigated. Several metabolic genes (DESUT3_29720, 35500, 33690, and 37760) were also found to be unique to the NIT-T3 genome; however, these genes are disconnected from other functional genes and do not complete a series of metabolic processes.
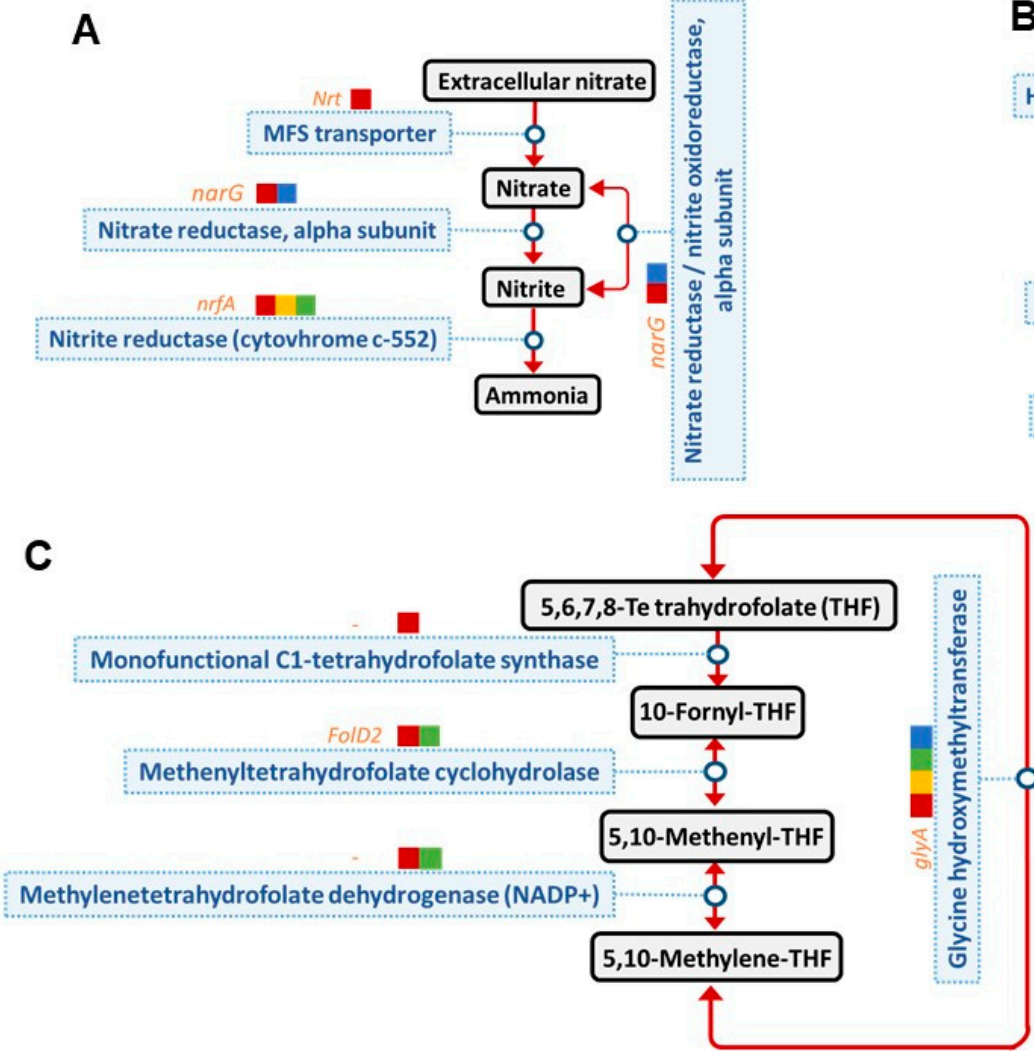

B

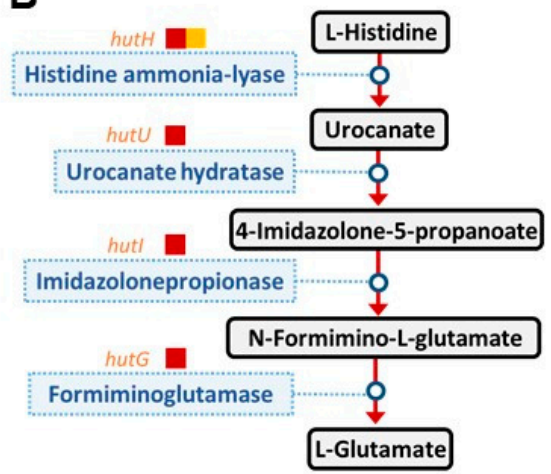

Figure 8. The complete metabolic pathway of strain NIT-T3 distinct from that of the complete genomes of three Desulfuromonas strains. Metabolic pathways of (A) nitrate reduction, (B) histidine degradation, and (C) C1-unit interconversion.

\section{Conclusions}

The isolation and polyphasic characterization of the novel strain NIT-T3 revealed an increase in the metabolic capability of the genus Desulfuromonas. In total, 79 of the large number of $c$-type cytochromes suggested their substantial role as representative electrogenic bacteria in natural and synthetic environments. The interswitching phylogeny of EET-related genes in members of the order Desulfuromonadales beyond the family suggested the early divergence and the substantial roles of the order Desulfuromonadales rather than the specific genus like Geobacter in EETs in various environments. Strain NIT-T3 is proposed as a new species of the genus Desulfuromonas according to the phenotype and phylotype and the description is provided as follows: 


\section{Description of Desulfuromonas versatilis sp. Nov.}

Desulfuromonas versatilis (ver.sa'ti.lis L. masc./fem. Adj. versatilis, versatile with respect to the capability to use a variety of electron donors and acceptors).

Cells are Gram-negative, non-spore-forming, rod-shaped, non-motile, and strictly anaerobic. Optimal growth was observed at $25^{\circ} \mathrm{C}, \mathrm{pH}$ 6.8-7.1, and $\mathrm{NaCl}$ concentration ranging from $0.2 \%$ to $1 \%$. The substrates used for nitrate reduction included hydrogen, formate, acetate, lactate, pyruvate, succinate, malate, isopropanol, peptone, and yeast extract. Elemental sulfur, iron oxide, fumarate, nitrate, AQDS, malate, graphite electrode, and GO served as terminal electron acceptors coupled to acetate oxidation. The major cellular fatty acids present were $C_{16: 1} \omega 7 c$ and $C_{16: 0}$, and the major respiratory quinone in the cell wall of NIT-T3 was MK-8. The type strain, NIT-T3 ${ }^{\mathrm{T}}$ was isolated from a mixture of seawater and coastal sand. The genomic $\mathrm{G}+\mathrm{C}$ content in the type strain was $63.1 \%$.

Supplementary Materials: The following are available online at https: / www.mdpi.com/article / 10.3390/microorganisms9091953/s1, Table S1: Genes uniquely present in strain T3 compared with other species of the genus Desulfuromonas.

Author Contributions: L.X.: Data analysis and writing; N.Y.: isolation, data curation, conceptualization, writing, supervision, funding acquisition; S.I., data analysis; L.M., data curation and data analysis. All authors have read and agreed to the published version of the manuscript.

Funding: This study received funding from MEXT/JSPS KAKENHI (grant numbers: 18K18876 and 16H06279) and the JSPS Joint Research Program with NSFC. The genome analysis was supported by MEXT KAKENHI (No. 221S0002).

Institutional Review Board Statement: Not applicable.

Informed Consent Statement: Not applicable.

Data Availability Statement: The data presented in this study are available on request from the corresponding author.

Acknowledgments: We would like to thank Tomomi Suzuki and Asuka Akita for their technical support in the cultivation and genomic experiments.

Conflicts of Interest: The authors declare no conflict of interest.

\section{References}

1. Logan, B.E.; Hamelers, B.; Rozendal, R.; Schröder, U.; Keller, J.; Freguia, S.; Aelterman, P.; Verstraete, W.; Rabaey, K. Microbial fuel cells: Methodology and technology. Environ. Sci. Technol. 2006, 40, 5181-5192. [CrossRef]

2. Zou, S.; He, Z. Efficiently "pumping out" value-added resources from wastewater by bioelectrochemical systems: A review from energy perspectives. Water Res. 2018, 131, 62-73. [CrossRef] [PubMed]

3. Wang, X.; Aulenta, F.; Puig, S.; Esteve-Núñez, A.; He, Y.; Mu, Y.; Rabaey, K. Microbial electrochemistry for bioremediation. Environ. Sci. Ecotechnol. 2020, 1, 100013. [CrossRef]

4. Zhi, W.; Ge, Z.; He, Z.; Zhang, H. Methods for understanding microbial community structures and functions in microbial fuel cells: A review. Bioresour. Technol. 2014, 171, 461-468. [CrossRef] [PubMed]

5. Saheb-Alam, S.; Persson, F.; Wilén, B.M.; Hermansson, M.; Modin, O. Response to starvation and microbial community composition in microbial fuel cells enriched on different electron donors. Microb. Biotechnol. 2019, 12, 962-975. [CrossRef] [PubMed]

6. Wu, H.; Yang, M.; Tsui, T.-H.; Yin, Z.; Yin, C. Comparative evaluation on the utilization of applied electrical potential in a conductive granule packed biotrickling filter for continuous abatement of xylene: Performance, limitation, and microbial community. J. Environ. Manag. 2020, 274, 111145. [CrossRef] [PubMed]

7. Katuri, K.P.; Kamireddy, S.; Kavanagh, P.; Mohammad, A.; Conghaile, P.; Kumar, A.; Saikaly, P.E.; Leech, D. Electroactive biofilms on surface functionalized anodes: The anode respiring behavior of a novel electroactive bacterium, Desulfuromonas acetexigens. bioRxiv 2020. [CrossRef]

8. Marone, A.; Carmona-Martínez, A.A.; Sire, Y.; Meudec, E.; Steyer, J.P.; Bernet, N.; Trably, E. Bioelectrochemical treatment of table olive brine processing wastewater for biogas production and phenolic compounds removal. Water Res. 2016, 100, 316-325. [CrossRef] [PubMed]

9. Zubchenko, L.; Kuzminskiy, Y. Characteristics of biofilm formation process in the bioelectrochemical systems, working in batch-mode of cultivation. Chem. Chem. Technol. 2017, 11, 105-110. [CrossRef] 
10. Kouzuma, A.; Ishii, S.; Watanabe, K. Metagenomic insights into the ecology and physiology of microbes in bioelectrochemical systems. Bioresour. Technol. 2018, 255, 302-307. [CrossRef]

11. Nijenhuis, I.; Nikolausz, M.; Köth, A.; Felföldi, T.; Weiss, H.; Drangmeister, J.; Großmann, J.; Kästner, M.; Richnow, H.H. Assessment of the natural attenuation of chlorinated ethenes in an anaerobic contaminated aquifer in the Bitterfeld/Wolfen area using stable isotope techniques, microcosm studies and molecular biomarkers. Chemosphere 2007, 67, 300-311. [CrossRef]

12. Dowideit, K.; Scholz-Muramatsu, H.; Miethling-Graff, R.; Vigelahn, L.; Freygang, M.; Dohrmann, A.B.; Tebbe, C.C. Spatial heterogeneity of dechlorinating bacteria and limiting factors for in situ trichloroethene dechlorination revealed by analyses of sediment cores from a polluted field site. FEMS Microbiol. Ecol. 2010, 71, 444-459. [CrossRef]

13. Chang, Y.H.; Cheng, T.W.; Lai, W.J.; Tsai, W.Y.; Sun, C.H.; Lin, L.H.; Wang, P.L. Microbial methane cycling in a terrestrial mud volcano in eastern Taiwan. Environ. Microbiol. 2012, 14, 895-908. [CrossRef]

14. Pfennig, N.; Biebl, H. Desulfuromonas acetoxidans gen. nov. and sp. nov., a new anaerobic, sulfur-reducing, acetate-oxidizing bacterium. Arch. Microbiol. 1976. [CrossRef] [PubMed]

15. Finster, K.; Bak, F.; Pfennig, N. Desulfuromonas acetexigens sp. nov., a dissimilatory sulfur-reducing eubacterium from anoxic freshwater sediments. Arch. Microbiol. 1994. [CrossRef]

16. Coates, J.D.; Lonergan, D.J.; Philips, E.J.P.; Jenter, H.; Lovley, D.R.; Lovley, D.R. Desulfuromonas palmitatis sp. nov., a marine dissimilatory Fe(ill) reducer that can oxidize long-chain fatty acids. Arch. Microbiol. 1995, 164, 406-413. [CrossRef] [PubMed]

17. Finster, K.; Coates, J.D.; Liesack, W.; Pfennig, N. Desulfuromonas thiophila sp. nov., a new obligately sulfur-reducing bacterium from anoxic freshwater sediment. Int. J. Syst. Bacteriol. 1997, 47, 754-758. [CrossRef] [PubMed]

18. Krumholz, L.R. Desulfuromonas chloroethenica sp. nov. uses tetrachloroethylene and trichloroethylene as electron acceptors. Int. J. Syst. Bacteriol. 1997, 47, 1262-1263. [CrossRef]

19. Sung, Y.; Ritalahti, K.M.; Sanford, R.A.; Urbance, J.W.; Flynn, S.J.; Tiedje, J.M.; Löffler, F.E. Characterization of two tetrachloroethene-reducing, acetate-oxidizing anaerobic bacteria and their description as Desulfuromonas michiganensis sp. nov. Appl. Environ. Microbiol. 2003, 69, 2964-2974. [CrossRef] [PubMed]

20. Vandieken, V.; Mußmann, M.; Niemann, H.; Jørgensen, B.B. Desulfuromonas svalbardensis sp. nov. and Desulfuromusa ferrireducens sp. nov., psychrophilic, Fe(III)-reducing bacteria isolated from Arctic sediments, Svalbard. Int. J. Syst. Evol. Microbiol. 2006, 56, 1133-1139. [CrossRef]

21. An, T.T.; Picardal, F.W. Desulfuromonas carbonis sp. nov., an Fe(III)-, S0- and Mn(IV)-reducing bacterium isolated from an active coalbed methane gas well. Int. J. Syst. Evol. Microbiol. 2015, 65, 1686-1693. [CrossRef]

22. Kim, S.; Park, S.; Cha, I.; Min, D.; Kim, J.; Chung, W.; Chae, J.; Jeon, C.O.; Rhee, S. Metabolic versatility of toluene-degrading, iron-reducing bacteria in tidal flat sediment, characterized by stable isotope probing-based metagenomic analysis. Environ. Microbiol. 2014, 16, 189-204. [CrossRef]

23. Guo, Y.; Aoyagi, T.; Inaba, T.; Sato, Y.; Habe, T.H.H. Complete Genome Sequence of Desulfuromonas sp. Strain AOP6, an Iron (III) Reducer Isolated from Subseafloor Sediment. Microbiol. Resour. Announc. 2020, 9, e01325-19. [CrossRef] [PubMed]

24. Zhang, T.; Bain, T.S.; Barlett, M.A.; Dar, S.A.; Snoeyenbos-West, O.L.; Nevin, K.P.; Lovley, D.R. Sulfur oxidation to sulfate coupled with electron transfer to electrodes by Desulfuromonas strain TZ1. Microbiology 2014, 160, 123-129. [CrossRef] [PubMed]

25. Badalamenti, J.P.; Summers, Z.M.; Chan, C.H.; Gralnick, J.A.; Bond, D.R. Isolation and genomic characterization of "Desulfuromonas soudanensis WTL", a metal- and electrode-respiring bacterium from anoxic deep subsurface brine. Front. Microbiol. 2016, 7, 1-11. [CrossRef] [PubMed]

26. Jacobsen, T.; Bardiaux, B.; Francetic, O.; Izadi-Pruneyre, N.; Nilges, M. Structure and function of minor pilins of type IV pili. Med. Microbiol. Immunol. 2020, 209, 301-308. [CrossRef] [PubMed]

27. Yoshida, N.; Goto, Y.; Miyata, Y.; Thakur, V.K. Selective growth of and electricity production by marine exoelectrogenic bacteria in self-aggregated hydrogel of microbially reduced graphene oxide. C. J. Carbon Res. 2016, 2016, 15. [CrossRef]

28. Yoshida, N.; Miyata, Y.; Doi, K.; Goto, Y.; Nagao, Y.; Tero, R.; Hiraishi, A. Graphene oxide-dependent growth and self-Aggregation into a hydrogel complex of exoelectrogenic bacteria. Sci. Rep. 2016, 6, 15. [CrossRef]

29. Goto, Y.; Yoshida, N.; Umeyama, Y.; Yamada, T.; Tero, R.; Hiraishi, A. Enhancement of electricity production by graphene oxide in soil microbial fuel cells and plant microbial fuel cells. Front. Bioeng. Biotechnol. 2015, 3, 42. [CrossRef] [PubMed]

30. Goto, Y.; Yoshida, N. Scaling up microbial fuel cells for treating. Water 2019, 11, 1803. [CrossRef]

31. Goto, Y.; Yoshida, N. Microbially reduced graphene oxide shows efficient electricity recovery from artificial dialysis wastewater. J. Gen. Appl. Microbiol. 2017, 63, 165-171. [CrossRef]

32. Yoshida, N.; Miyata, Y.; Mugita, A.; Iida, K. Electricity recovery from municipal sewage wastewater using a hydrogel complex composed of microbially reduced graphene oxide and sludge. Materials 2016, 9, 742. [CrossRef] [PubMed]

33. Yoshida, N.; Asahi, K.; Sakakibara, Y.; Miyake, K.; Katayama, A. Isolation and quantitative detection of tetrachloroethene (PCE)-dechlorinating bacteria in unsaturated subsurface soils contaminated with chloroethenes. J. Biosci. Bioeng. 2007, 104, 91-97. [CrossRef] [PubMed]

34. Ismaeil, M.; Yoshida, N.; Katayama, A. Bacteroides sedimenti sp. nov., isolated from a chloroethenes-dechlorinating consortium enriched from river sediment. J. Microbiol. 2018, 56, 619-627. [CrossRef] [PubMed]

35. Nagahashi, W.; Yoshida, N. Comparative evaluation of fibrous artificial carbons and bamboo charcoal in terms of recovery of current from sewage wastewater. J. Gen. Appl. Microbiol. 2021, in press. [CrossRef] 
36. Tamaoka, J.; Katayama-Fujimura, Y.; Kuraishi, H. Analysis of bacterial menaquinone mixtures by high performance liquid chromatography. J. Appl. Bacteriol. 1983, 54, 31-36. [CrossRef]

37. Ismaeil, M.; Yoshida, N.; Katayama, A. Identification of multiple dehalogenase genes involved in tetrachloroethene-to-ethene dechlorination in a dehalococcoides-dominated enrichment culture. Biomed. Res. Int. 2017, 2017, 9191086. [CrossRef] [PubMed]

38. Tanizawa, Y.; Fujisawa, T.; Nakamura, Y. DFAST: A flexible prokaryotic genome annotation pipeline for faster genome publication. Bioinformatics 2018, 34, 1037-1039. [CrossRef]

39. Overbeek, R.; Olson, R.; Pusch, G.D.; Olsen, G.J.; Davis, J.J.; Disz, T.; Edwards, R.A.; Gerdes, S.; Parrello, B.; Shukla, M.; et al. The SEED and the rapid annotation of microbial genomes using Subsystems Technology (RAST). Nucleic Acids Res. 2014, 42, 206-214. [CrossRef]

40. Kumar, S.; Stecher, G.; Li, M.; Knyaz, C.; Tamura, K. MEGA X: Molecular evolutionary genetics analysis across computing platforms. Mol. Biol. Evol. 2018, 35, 1547-1549. [CrossRef] [PubMed]

41. Meier-Kolthoff, J.P.; Göker, M.; Spröer, C.; Klenk, H.P. When should a DDH experiment be mandatory in microbial taxonomy? Arch. Microbiol. 2013, 195, 413-418. [CrossRef]

42. Kim, M.; Oh, H.S.; Park, S.C.; Chun, J. Towards a taxonomic coherence between average nucleotide identity and $16 \mathrm{~S}$ rRNA gene sequence similarity for species demarcation of prokaryotes. Int. J. Syst. Evol. Microbiol. 2014, 64, 346-351. [CrossRef] [PubMed]

43. Sun, D.; Wang, A.; Cheng, S.; Yates, M.; Logan, B.E. Geobacter anodireducens sp. nov., an exoelectrogenic microbe in bioelectrochemical systems. Int. J. Syst. Evol. Microbiol. 2014, 64, 3485-3491. [CrossRef] [PubMed]

44. Viulu, S.; Nakamura, K.; Okada, Y.; Saitou, S.; Takamizawa, K. Geobacter luticola sp. nov., an Fe(III)-reducing bacterium isolated from lotus field mud. Int. J. Syst. Evol. Microbiol. 2013, 63, 442-448. [CrossRef]

45. Shelobolina, E.S.; Vrionis, H.A.; Findlay, R.H.; Lovley, D.R. Geobacter uraniireducens sp. nov., isolated from subsurface sediment undergoing uranium bioremediation. Int. J. Syst. Evol. Microbiol. 2008, 58, 1075-1078. [CrossRef] [PubMed]

46. Shelobolina, E.S.; Nevin, K.P.; Blakeney-Hayward, J.D.; Johnsen, C.V.; Plaia, T.W.; Krader, P.; Woodard, T.; Holmes, D.E.; VanPraagh, C.G.; Lovley, D.R. Geobacter pickeringii sp. nov., Geobacter argillaceus sp. nov. and Pelosinus fermentans gen. nov., sp. nov., isolated from subsurface kaolin lenses. Int. J. Syst. Evol. Microbiol. 2007, 57, 126-135. [CrossRef]

47. Hedrick, D.B.; Peacock, A.D.; Lovley, D.R.; Woodard, T.L.; Nevin, K.P.; Long, P.E.; White, D.C. Polar lipid fatty acids, LPS-hydroxy fatty acids, and respiratory quinones of three Geobacter strains, and variation with electron acceptor. J. Ind. Microbiol. Biotechnol. 2009, 36, 205-209. [CrossRef] [PubMed]

48. Kunapuli, U.; Jahn, M.K.; Lueders, T.; Geyer, R.; Heipieper, H.J.; Meckenstock, R.U. Desulfitobacterium aromaticivorans sp. nov. and Geobacter toluenoxydans sp. nov., iron-reducing bacteria capable of anaerobic degradation of monoaromatic hydrocarbons. Int. J. Syst. Evol. Microbiol. 2010, 60, 686-695. [CrossRef]

49. Chen, C.; Chen, H.; Zhang, Y.; Thomas, H.R.; Frank, M.H.; He, Y. TBtools: An integrative toolkit developed for interactive analyses of big biological data. Mol. Plant. 2020, 13, 1194-1202. [CrossRef]

50. Sigrist, C.J.A.; Cerutti, L.; Hulo, N.; Gattiker, A.; Falquet, L.; Pagni, M.; Bairoch, A.; Bucher, P. PROSITE: A documented database using patterns and profiles as motif descriptors. Brief. Bioinform. 2002, 3, 265-274. [CrossRef]

51. Jiang, Y.; Shi, M.; Shi, L. Molecular underpinnings for microbial extracellular electron transfer during biogeochemical cycling of earth elements. Sci. China Life Sci. 2019, 62, 1275-1286. [CrossRef] [PubMed]

52. Levar, C.E.; Chan, C.H.; Mehta-Kolte, M.G.; Bond, D.R. An inner membrane cytochrome required only for reduction of high redox potential extracellular electron acceptors. MBio 2014, 5, e02034-14. [CrossRef]

53. Levar, C.E.; Hoffman, C.L.; Dunshee, A.J.; Toner, B.M.; Bond, D.R. Redox potential as a master variable controlling pathways of metal reduction by Geobacter sulfurreducens. ISME J. 2017, 11, 741-752. [CrossRef]

54. Morgado, L.; Brulx, M.; Pessanha, M.; Londer, Y.Y.; Salgueiro, C.A. Thermodynamic characterization of a triheme cytochrome family from geobacter sulfurreducens reveals mechanistic and functional diversity. Biophys. J. 2010, 99, 293-301. [CrossRef] [PubMed]

55. Liu, Y.; Fredrickson, J.K.; Zachara, J.M.; Shi, L. Direct involvement of ombB, omaB, and omcB genes in extracellular reduction of Fe (III) by Geobacter sulfurreducens PCA. Front. Microbiol. 2015, 6, 1075. [CrossRef]

56. Liu, Y.; Wang, Z.; Liu, J.; Levar, C.; Edwards, M.J.; Babauta, J.T.; Kennedy, D.W.; Shi, Z.; Beyenal, H.; Bond, D.R.; et al. A trans-outer membrane porin-cytochrome protein complex for extracellular electron transfer by Geobacter sulfurreducens PCA. Environ. Microbiol. Rep. 2014, 6, 776-785. [CrossRef] [PubMed]

57. Otero, F.J.; Chan, C.H.; Bond, D.R. Identification of different putative outer membrane electron conduits necessary for Fe(III) citrate, $\mathrm{Fe}(\mathrm{III})$ oxide, $\mathrm{Mn}(\mathrm{IV})$ oxide, or electrode reduction by Geobacter sulfurreducens. J. Bacteriol. 2018, 200, e00347-18. [CrossRef]

58. Trumpower, B. The protonmotive Q cycle. J. Biol. Chem. 1990, 265, 11409-11412. [CrossRef]

59. Mollaei, M.; Timmers, P.H.A.; Suarez-Diez, M.; Boeren, S.; van Gelder, A.H.; Stams, A.J.M.; Plugge, C.M. Comparative proteomics of Geobacter sulfurreducens PCAT in response to acetate, formate and/or hydrogen as electron donor. Environ. Microbiol. 2021, 23, 299-315. [CrossRef]

60. Seidel, J.; Hoffmann, M.; Ellis, K.E.; Seidel, A.; Spatzal, T.; Gerhardt, S.; Elliott, S.J.; Einsle, O. MacA is a second cytochrome c peroxidase of Geobacter sulfurreducens. Biochemistry 2012, 51, 2747-2756. [CrossRef]

61. Aklujkar, M.; Coppi, M.V.; Leang, C.; Kim, B.C.; Chavan, M.A.; Perpetua, L.A.; Giloteaux, L.; Liu, A.; Holmes, D.E. Proteins involved in electron transfer to Fe (III) and Mn (IV) oxides by Geobacter sulfurreducens and Geobacter uraniireducens. Microbiology 2013, 159, 515-535. [CrossRef] 
62. Butler, J.E.; Young, N.D.; Lovley, D.R. Evolution of electron transfer out of the cell: Comparative genomics of six Geobacter genomes. BMC Genom. 2010, 11, 40. [CrossRef]

63. Zheng, S.; Liu, F.; Li, M.; Xiao, L.; Wang, O. Comparative transcriptomic insights into the mechanisms of electron transfer in Geobacter co-cultures with activated carbon and magnetite. Sci. China Life Sci. 2018, 61, 787-798. [CrossRef] [PubMed]

64. Craig, L.; Forest, K.T.; Maier, B. Type IV pili: Dynamics, biophysics and functional consequences. Nat. Rev. Microbiol. 2019, 17, 429-440. [CrossRef] [PubMed]

65. Lovley, D.R.; Walker, D.J.F. Geobacter protein nanowires. Front. Microbiol. 2019, 10, 2078. [CrossRef]

66. Lovley, D.R. Electrically conductive pili: Biological function and potential applications in electronics. Curr. Opin. Electrochem. 2017, 4, 190-198. [CrossRef]

67. Walker, D.J.; Adhikari, R.Y.; Holmes, D.E.; Ward, J.E.; Woodard, T.L.; Nevin, K.P.; Lovley, D.R. Electrically conductive pili from pilin genes of phylogenetically diverse microorganisms. ISME J. 2018, 12, 48-58. [CrossRef]

68. Herzberg, M.; Kaye, I.K.; Peti, W.; Wood, T.K. YdgG (TqsA) controls biofilm formation in Escherichia coli K-12 through autoinducer 2 transport. J. Bacteriol. 2006, 188, 587-598. [CrossRef]

69. Button, J.E.; Silhavy, T.J.; Ruiz, N. A suppressor of cell death caused by the loss of $\sigma$ E downregulates extracytoplasmic stress responses and outer membrane vesicle production in Escherichia coli. J. Bacteriol. 2007, 189, 1523-1530. [CrossRef] 